\title{
Acupuncture Randomized Trials (ART) in Patients with Chronic Low Back Pain and Osteoarthritis of the Knee - Design and Protocols
}

\author{
$\begin{array}{lll}\text { B. Brinkhaus } & \mathrm{a}^{*} & \text { C. Becker-Witt } \\ \mathrm{a}^{*}\end{array}$ \\ S. Jena ${ }^{a} \quad K$ Linde $^{b} \quad$ A. Streng ${ }^{b}$ \\ S. Wagenpfeil ${ }^{\mathrm{C}}$ \\ D. Irnich ${ }^{d}$ J. Hummelsberger ${ }^{\mathrm{e}}$ \\ D. Melchart ${ }^{b, f}$ S.N. Willich ${ }^{a}$
}

a Institut für Sozialmedizin, Epidemiologie und Gesundheitsökonomie, Klinikum Charité, Humboldt-Universität zu Berlin

b Zentrum für naturheilkundliche Forschung, II. Medizinische Klinik und Poliklinik, Technische Universität München

${ }^{\mathrm{c}}$ Institut für Medizinische Statistik und Epidemiologie, Technische Universität München

${ }^{\mathrm{d}}$ Klinik für Anästhesiologie, Ludwig-Maximilians-Universität München

e Internationale Gesellschaft für Chinesische Medizin (Societas Medicinae Sinensis, SMS), München

${ }^{f}$ Abteilung Naturheilkunde, Department für Innere Medizin, Universitätsspital Zürich

${ }^{*}$ Contributed equally.

Key Words

Acupuncture - Traditional Chinese Medicine .

Low back pain · Osteoarthritis · Randomized controlled trial

\section{Summary}

Background: We report on the study design and protocols of two randomized controlled trials (Acupuncture Randomized Trials = ART) that investigate the efficacy of acupuncture in the treatment of chronic low back pain and osteoarthritis of the knee, respectively. Objective: To investigate whether acupuncture is more efficacious than (a) no treatment or (b) minimal acupuncture in the treatment of low back pain and osteoarthritis. Design: Two randomized, controlled, multicenter trials with three treatment arms and a total follow-up time of 52 weeks. Setting: 30 practitioners and outpatient units in Germany specialized in acupuncture treatment. Patients: 300 patients will be included in each study. In the low back pain trial, patients will be included according to clinical diagnosis. In the osteoarthritis pain trial, patients will be included according to the American College of Rheumatology criteria. Interventions: Patients are randomly assigned to receive either (1) semi-standardized acupuncture (150 patients), (2) minimal acupuncture at non-acupuncture points (75 patients), or (3) no treatment for two months followed by semi-standardized acupuncture (75 patients, waiting list control). Acupuncture treatment consists of 12 sessions per patient over a period of 8 weeks. Main Outcome Measure: The main outcome measure is the difference between baseline and the end of the 8-week treatment period in the following parameters: pain intensity as measured by a visual analogue scale (VAS; 0-100 mm) in the low back pain trial and by the Western Ontario and McMaster Universities Osteoarthritis Score (WOMAC) in the osteoarthritis trial. Outlook: The results of these two studies (available in 2004) will provide health care providers and policy makers with the information needed to make scientifically sound assessments of acupuncture therapy.

\section{Schlüsselwörter}

Akupunktur · Traditionelle Chinesische Medizin . Lendenwirbelsäulen-Schmerzen · Gonarthrose · Randomisierte klinische Studie

\section{Zusammenfassung}

Hintergrund: Wir berichten über das Studiendesign und essentielle Teile der Protokolle zweier randomisierter Studien (“Acupuncture Randomized Trials" = ART) bei Patienten mit chronischen Schmerzen im Bereich der Lendenwirbelsäule (LWS) und Gonarthrose. Ziel: Ziel der Studien ist es, zu untersuchen, ob eine Serie von Akupunkturbehandlungen in der Therapie von chronischen Schmerzen im Bereich der LWS bzw. bei Gonarthrose wirksamer ist als (a) keine Therapie (Wartelistenkontrolle) bzw. (b) eine Minimalakupunktur. Design: Zwei randomisierte, kontrollierte, dreiarmige Multizenterstudien mit einem Follow-up von 52 Wochen. Prüfzentren: 30 auf Akupunktur spezialisierte Arztpraxen und Ambulanzen in Deutschland. Patienten: Pro Studie 300 Patienten mit der klinischen Diagnose chronische Schmerzen im Bereich der LWS oder Gonarthrose definiert nach den Kriterien des American College of Rheumatology. Interventionen: Patienten erhalten randomisiert entweder (a) eine semistandardisierte Akupunkturbehandlung (150 Patienten), (b) eine Minimalakupunktur an Nicht-Akupunkturpunkten (75 Patienten) oder (c) keine Behandlung für 2 Monate, gefolgt von einer semistandardisierten Akupunkturbehandlung (75 Patienten, Wartelistengruppe). Die Akupunkturintervention erfolgt in 12 Akupunktursitzungen über einen Zeitraum von 8 Wochen. Hauptzielkriterien: Hauptzielkriterium ist die Veränderung folgender Parameter im Vergleich vor bzw. nach Abschluss der Studienintervention (8 Wochen nach Randomisierung): Schmerzscore gemessen anhand der visuellen Analogskala (VAS; 0-100 mm) in der LWSSchmerz-Studie und Western Ontario and McMaster Universities Osteoarthritis (WOMAC)-Score in der Gonarthrose-Studie. Ausblick: Erste Studienergebnisse, die eine Basis für eine wissenschaftliche und gesundheitspolitische Neubeurteilung der Akupunktur bieten werden, sind im Jahr 2004 zu erwarten.

\begin{tabular}{ll}
\hline KARGER & ( ) 2003 S. Karger GmbH, Freiburg \\
Fax +49 7614520714 & Accessible online at: \\
$\begin{array}{l}\text { E-mail Information@Karger.de } \\
\text { www.karger.com }\end{array}$ & www.karger.com/fkm \\
&
\end{tabular}

Dr. Benno Brinkhaus

Institut für Sozialmedizin, Epidemiologie und Gesundheitsökonomie, Klinikum Charité,

Humboldt-Universität zu Berlin

D-10098 Berlin

Tel. +49 30450 529-002, Fax -902

E-mail benno.brinkhaus@charite.de 


\section{Introduction}

Throughout the 1990s, the costs of acupuncture therapy administered by physicians were partially covered by the German health insurance funds on an informal basis. Under increasing pressure to cut health care costs, however, the Federal Committee of Physicians and Health Insurers ('Bundesausschuss der Ärzte und Krankenkassen') decided in October 2000 that the scientific evidence supporting acupuncture was insufficient to justify routine reimbursement. Nevertheless, it recommended that special Model Projects on Acupuncture ('Modellvorhaben Akupunktur') be developed in order to determine the role of acupuncture in the treatment of certain illnesses. In particular, the Committee requested that 'sham'-controlled, randomized clinical trials be conducted on the role of acupuncture in the management of chronic low back pain, chronic pain associated with osteoarthritis, and chronic headache [1] - all conditions for which the available evidence had shown acupuncture to be a promising means of treatment. Today, the costs of acupuncture therapy can be covered by the health insurance funds for a limited period of time on a nation-wide level, provided that the treatment be administered within the framework of Model Projects which also include 'sham'-controlled, randomized trials as part of the evaluation strategy.

In this paper, we report on the design and protocols of two such clinical trials, which are part of a large acupuncture research initiative (the 'Modellvorhaben der Techniker Krankenkasse und der dem Modellvorhaben beigetretenen Krankenkassen' and the 'Modellvorhaben der Ersatzkassen'). One of the trials investigates the efficacy of acupuncture in the treatment of chronic low back pain. The other trial examines whether acupuncture is effective in the treatment of osteoarthritis of the knee. In another, separate paper [2], we present details on clinical trials that examine the role of acupuncture in the management of migraine and tension-type headache.

\section{State of Research on Acupuncture for Low Back Pain}

To date, the few randomized clinical trials to investigate the efficacy of acupuncture in the treatment of back pain have been evaluated in three systematic reviews $[3,5,6]$. Interestingly, although the material analyzed is relatively uniform, the authors of each of the three systematic reviews come to different conclusions.

The first review is a meta-analysis of 9 randomized controlled trials, each of which investigated the use of some form of acupuncture in the treatment of back pain. Although the authors conclude that acupuncture was superior to various control interventions in these trials, they point out that there was not enough evidence to determine whether acupuncture was superior to placebo [3]. The update of this meta-analysis supports the above-mentioned conclusion [4].

In the second review, 11 randomized controlled trials were evaluated in order to determine the efficacy of acupuncture in the treatment of non-specific low back pain [5]. The evidence summarized in this review indicates that acupuncture is not efficacious in the treatment of low back pain. More specifically, 8 of the 11 trials showed that there was limited evidence that acupuncture was not more efficacious than placebo or 'sham' acupuncture. In the 3 remaining trials - all of which were methodologically problematic - comparisons between acupuncture and no treatment yielded conflicting results. However, 2 of the trials indicated that acupuncture seemed to be no more effective than trigger point injections or transcutaneous electric nerval stimulation (TENS).

Finally, in the third review, 13 randomized controlled trials investigating the efficacy of acupuncture in the treatment of chronic neck and back pain were analyzed. The authors of this review conclude that there is no convincing evidence for the analgesic efficacy of acupuncture in this context [6].

Since the completion of the systematic review several new trials have been published [7-10] which, however, did not change the overall evidence picture fundamentally.

\section{State of Research on Acupuncture for Osteoarthritis of the Knee}

A certain amount of confusion in the literature has been caused by the inconsistent use of the term 'osteoarthritis' in English and in German. In German, the term 'Osteoarthrose' or 'Gonarthrose' is used to describe the degenerative aspect of the disease. In English, on the other hand, the term 'osteoarthritis' is used to describe the clinical symptoms of the disease [11]. In the present paper, we use the term 'osteoarthritis' in the Anglo-American sense of the word.

A systematic review published in 2001 included 7 randomized controlled trials representing 393 patients with osteoarthritis of the knee [12]. The authors found strong evidence that acupuncture was more effective than 'sham' acupuncture in terms of pain reduction. With regard to improvements in function, however, the evidence was found to be inconclusive. There was also insufficient evidence for the authors to determine whether the efficacy of acupuncture was similar to that of other treatments.

Since the completion of the systematic review a non-rondomized trial has been published [13], in which the acupuncture group showed improved pain and function in patients with osteoarthritis of the knee.

\section{Aim of the Study}

The primary objective of the two trials presented in this paper is to investigate whether a semi-standardized acupuncture intervention is more effective than (a) no treatment or (b) a standardized minimal acupuncture intervention of 8 weeks of treatment for patients suffering either from chronic low back pain or pain associated with osteoarthritis of the knee. Secondary objectives include an assessment of long-term effectiveness and medical care over a period of 52 weeks.
Brinkhaus/Becker-Witt/Jena/Linde/Streng/ Wagenpfeil/Irnich/Hummelsberger/Melchart/ Willich 


\section{Patients and Methods}

\section{Design}

Both studies are randomized, three-armed multicenter trials comparing (1) a semi-standardized acupuncture treatment, (2) minimal acupuncture treatment, and (3) a no treatment (waiting list) condition (fig. 1). Patients are blinded to treatment in the acupuncture and minimal acupuncture arms of the studies. The total follow-up study period per patient is 52 weeks. After randomization, patients in the acupuncture and minimal acupuncture groups receive 12 treatment sessions over a period of 8 weeks. In the waiting list group, patients do not receive acupuncture for the first 8 weeks. After 8 weeks, patients in the waiting list group receive 12 acupuncture treatments over a period of 8 weeks. The acupuncture treatment administered in the waiting list group is the same as that given in the group treated with a semi-standardized acupuncture regimen.

The studies are performed according to the principles of the Declaration of Helsinki (Version Edinburgh 2000, cf. http://www.wits.ac.za/bioethics/ helsinki.htm). The protocols have been approved by the local ethics review boards in all regions where the study is being conducted. The study participants are insured according to the German law for medicinal products ('Medizinproduktegesetz').

Randomization into the 3 study arms is performed centrally by the Institute of Medical Statistics and Epidemiology (IMSE) at the Technische Universität München using the software Samp Size 2.0 [14]. Participating practitioners and outpatient units are not involved in the randomization process. Patients who meet the inclusion criteria and give written and oral consent are included in the study. After a patient is included in the study, his or her physician phones IMSE, where the patient is registered. Then the physician receives information from the IMSE regarding patient allocation both via phone and fax. This procedure assures that randomization cannot be influenced by the treating physicians.

\section{Patients}

Both ART studies aim to recruit a total of 300 patients each. Recruitment for the trials started in April 2002

For inclusion in the low back pain study, patients must meet the following criteria: age 40-75 years; clinical diagnosis of chronic low back pain with a disease duration of more than 6 months; pain intensity $\geq 40$ on a $100-\mathrm{mm}$ visual analogue scale (VAS) in the previous 7 days; only use of oral nonsteroidal anti-inflammatory drugs (NSAIDs) for pain treatment in the 4 weeks before treatment; written consent.

The main exclusion criteria for patients in the low back pain study are one or more of the following symptoms or diseases: protusio or prolapse of one or more intervertebral disc(s) with concurrent neurological symptoms; prior vertebral column surgery; infectious spondylopathy; low back pain caused by inflammatory, malignant, or autoimmune disease; congenital deformation of spine except slight lordosis or scoliosis; compression fracture caused by osteoporosis; spinal stenosis; spondylolysis or spondylolisthesis; condition warrants treatment with moxibustion; application for pension or disability benefits.

For inclusion in the osteoarthritis of the knee study, patients must meet the following criteria: age 50-75 years; diagnosis of osteoarthrosis according to the American College of Rheumatology (ACR) criteria; documented radiologic alterations in knee $\geq$ grade $1-2$ according to the KellgrenLawrence criteria [15, 16]; pain intensity of $\geq 40$ on a 100 -mm VAS in the previous 7 days; only use of oral NSAIDs for pain treatment in the 4 weeks before treatment; written consent.

Main exclusion criteria for patients in the osteoarthritis of the knee study are one or more of the following diseases or conditions: knee pain caused by inflammatory, malignant, or autoimmune disease; other reasons for knee pain, such as serious valgus/varus-defective position; prior knee surgery; arthroscopy of affected knee in the last year; chondroprotectiva or intra-articular injection in the last 4 months; systemic corticoid therapy or beginning of a new therapy for osteoarthritis in the last 4 weeks; local antiphlogistic therapy; application for pension or disability benefits.
Additional exclusion criteria for both ART studies are: acupuncture treatment during the last 12 months, physiotherapy and other treatments for low back pain and osteoarthrosis pain of the knee (with the exception of NSAIDs) during the previous 4 weeks; serious acute or chronic organic disease or mental disorder; pregnancy or breast feeding; blood coagulation disorder; current use of anticoagulants.

\section{Participating Physicians}

Participating trial physicians were recruited in a manner designed to ensure that their qualification is at least equal to the average qualification of physicians currently accredited for providing acupuncture in the German 'Modellvorhaben Akupunktur'. Physicians were thus required to fulfil all of the following criteria: (1) acupuncture training at least equivalent to an 'A-diploma' from one of the major German acupuncture societies (140 hours of acupuncture training); (2) all physicians had to have at least 3 years of practical experience with acupuncture; (3) all physicians had to participate in study training sessions on the trial methods, the interventions tested, and standards for performing clinical trials (ICH-GCP); (4) $50 \%$ of trial physicians had to have at least a 'B-diploma' ( 350 hours; about $20 \%$ of physicians accredited to provide acupuncture as part of the 'Modellvorhaben Akupunktur' and outside the trials have this qualification); (5) $50 \%$ had to have experience working in clinical studies. About 30 study centers are currently participating in the studies. Non-medical acupuncturists were excluded from the studies.

\section{Interventions}

The treatment strategies for acupuncture and minimal acupuncture were developed in a consensus process with experienced acupuncture experts (Hammes M, Hummelsberger J, Irnich D) representing the following two major German societies for medical acupuncture: German Medical Acupuncture Association ('Deutsche Ärztegesellschaft für Akupunktur', DÄGfA), Munich; International Society for Chinese Medicine (Societas Medicinae Sinensis, SMS), Munich. In a first step the three experts developed a proposal which was then presented to more than 30 experts (listed in 'Acknowledgments') from both acupuncture societies for discussion. The final strategies were defined by the three experts together with the study team and communicated to the external advisors. The final strategies were generally considered as a pragmatic compromise between the need for some standardization and the need for individualization.

Both the acupuncture and minimal acupuncture treatments consist of 12 sessions of 30 min duration, each administered over a period of 8 weeks (preferably 2 sessions in each of the first 4 weeks, followed by 1 session per week in the remaining 4 weeks). Patients in the waiting list group do not receive acupuncture treatment for a period of 8 weeks after randomization, after which they receive the acupuncture treatment described below.

Acupuncture treatment is semi-standardized (tables 1,2): All patients have to be treated with a selection of permitted local and distant points which must be selected according to the principles of Traditional Chinese Medicine by experienced acupuncturists. In addition, acupuncturists in both studies can use additional points (including ear acupuncture points or trigger points). The number and name of additional acupuncture points must be documented. A traditional Chinese syndrome diagnosis is requested, but not mandatory. If it is performed, the diagnosis must be documented. Needle length and diameter are not predefined but have to be documented. An irradiating needling sensation ('de qi') should be achieved if possible. Needles should be stimulated manually at least once in each session.

Number, duration, and frequency of the sessions in the minimal acupuncture group are the same as for the acupuncture group. In both studies, physicians are required to choose points for minimal acupuncture treatment out of a selection of 10 minimal acupuncture (MA) points (table 3). For the study on low back pain, we require that at least 6 out of 10 proposed points at non-classical sites be needled bilaterally (at least 12 needles altogether) in each session. In the osteoarthritis trial, the number of 
Fig. 1. Trial design, time schedule, and outcome parameters. Outcome parameters: visual analogue scale (VAS), Western Ontario and McMaster Universities Osteoarthritis Score (WOMAC), functional status measured by the 'Funktionsfragebogen HannoverRücken' (FFbH-R), the German version of the Pain Disability Index (PDI), scale for assessing emotional aspects of pain ('Schmerzempfindungs-Skala', SES), depression scale (ADS), the German version of the SF-36 to assess healthrelated quality of life $(\mathrm{SF}-36){ }^{*}=$ Osteoarthritis; ${ }^{* *}=$ LBP.

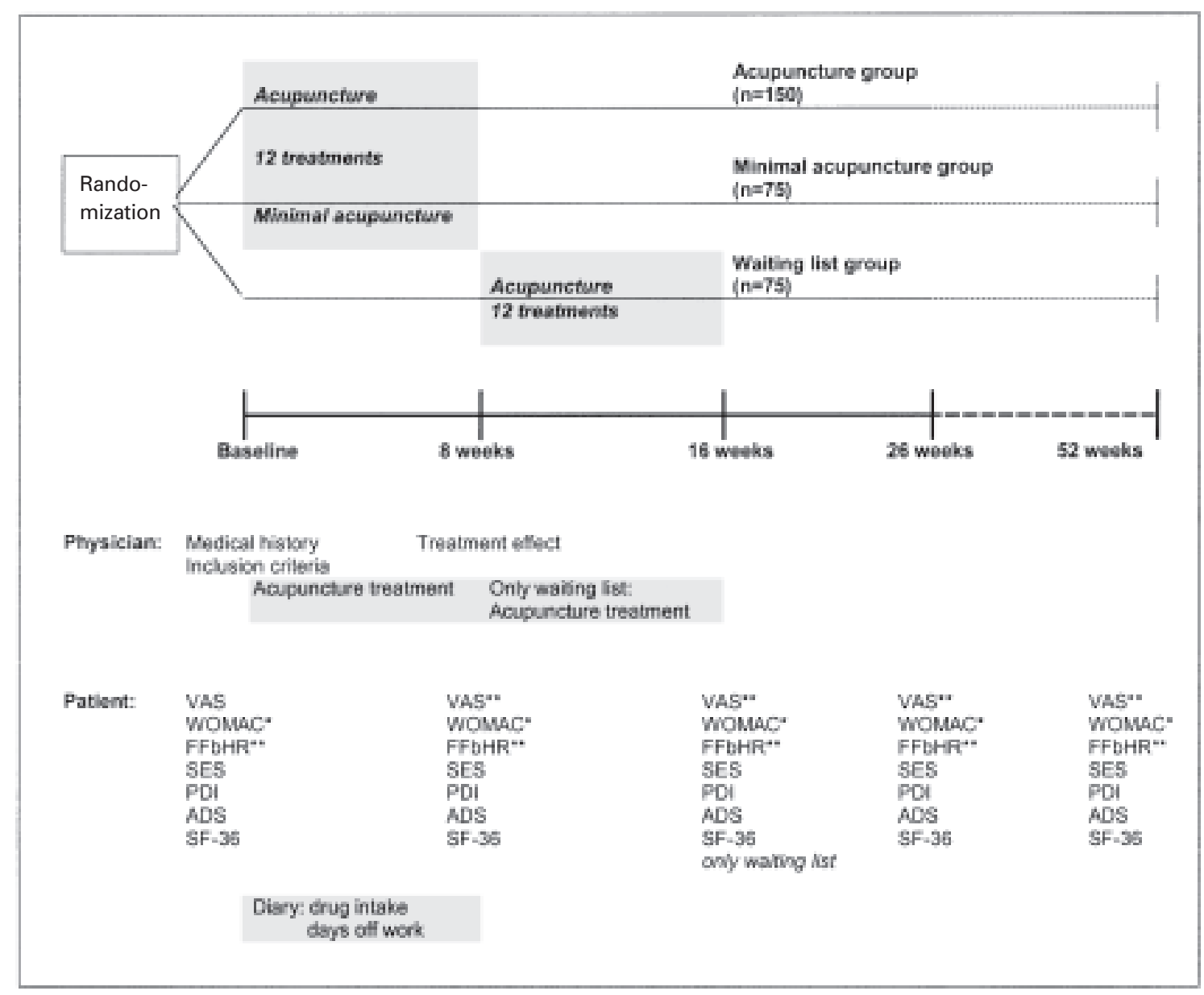

acupoints will be as follows: In the case of a bilateral osteoarthrosis, both knees have to be acupunctured with at least 8 out of 10 proposed points at non-classical sites (at least 16 needles altogether), whereas in the case of unilateral osteoarthrosis, the physician can choose uni- or bilateral acupuncture. In the case of unilateral acupuncture, the treatment will be performed with at least 8 needles.

Superficial insertion using fine needles $(20-40 \mathrm{~mm}$ in length) is recommended. 'De qi' and manual stimulation of the needles should be avoided; the needles should be placed subcutaneously. All acupuncturists were trained to apply minimal acupuncture and received a videotape and a brochure showing detailed information on minimal acupuncture.

The location of points was performed on the basis of individual body size using measuring units equal to the transverse width of finger (TF), or 'cun'. One cun is defined according to the traditional rules as the width of the interphalangeal joint of patient's thumb.

In both studies, patients are allowed to treat low back pain and osteoarthrosis of the knee, if required, with oral NSAIDs. If necessary, patients should continue chronic NSAID intake at the same dosage as before. In the first 8 weeks (acupuncture and minimal acupuncture group), or in the first 16 weeks (waiting list group), any concomitant pain medication is to be documented in a diary by the patients. In the 26 and 52 weeks follow-up period, patients will be asked retrospectively as to their medication intake. In particular, the use of other pain therapies, such as drugs acting through the central nervous system or corticosteroids, are prohibited.

Patients are informed in the study as follows: 'In this study, different types of acupuncture will be compared. One type is similar to the acupuncture treatment used in China. The other type does not follow these principles, but has also been associated with positive outcomes in clinical studies.'

\section{Outcome Measuremen}

For evaluating the main and secondary outcome measures, patients in both studies are requested to fill in a questionnaire containing the instruments mentioned below before randomization and at the end of weeks 8 , 26 , and 52 (i.e. following initiation of treatment). Patients in the waiting list group are asked to complete an additional questionnaire at the end of 16 weeks.

For the low back pain trial the main outcome measure is the difference in pain intensity measured by a VAS [17] $(0-100 \mathrm{~mm})$ in the week before randomization and again at the end of week 8 (waiting list group: week 16). Secondary outcome measures are changes in the following parameters between the week before randomization and at the end of weeks 8 , 26 , and 52: VAS at the end of weeks 26 and 52, functional status measured by the 'Funktionsfragebogen Hannover-Rücken' (FFbH-R) [18], and further secondary outcome parameters described below.

For the osteoarthritis trial the main outcome parameter is the difference between the Western Ontario and McMaster Universities Osteoarthritis Scores (WOMAC) [19-21] before randomization and at the end of week 8 (waiting list group: weeks 16).

The differences of the WOMAC score between the week before randomization and weeks 26 and 52 are secondary outcome parameters.

Patients are asked to fill in a modified version of the pain questionnaire designed by the German Society for the Study of Pain (DGSS; http://www.medizin.uni- koeln.de/projekte/dgss/Schmerzfragebogen.html) before treatment and at the end of weeks 8,26 , and 52. In addition to a number of questions on sociodemographic characteristics, numerical rating scales for pain intensity, questions on workdays lost, global assessments, etc., the DGSS pain questionnaire includes the following validated instruments: (1) the German version of the Pain Disability Index (PDI) 
Table 1. Acupuncture points used in low back pain trial

\section{Local points}

Choice of at least 4 local acupoints, insert bilaterally, from the following sample:

Bladder (BL) 20, BL 21, BL 22, BL 23, BL 24, BL 25, BL 26, BL 27 ,

BL 28, BL 29, BL 30, BL 31, BL 32, BL 33, BL 34, BL 50, BL 51,

BL 52, BL 53, BL 54

Gallbladder (GB) 30

Du Mai - Governing vessel (DU) 3, DU 4, DU 5, DU 6

Extrapoint (EX) Huatojiaji; EX Shiqizhuixia

\section{Distant points}

Additional choice of at least 2 local acupoints, insert bilaterally, from the following permitted sample:

Small intestine (SI) 3

BL 40, BL 60, BL 62

Kidney (KID) 3, KID 7

GB 31, GB 34, Gb 41

Liver (LIV) 3

DU 14, DU 20

In the event that there is a local or pseudoradicular radiate sensation, at least 2 local acupoints should be added.

Additional points (body and microsystem acupuncture) can be chosen. The total number of needles must not exceed 20 .

Table 2. Acupuncture points used in osteoarthrosis trial

\section{Local points}

Choice of at least 6 local acupoints from the following sample:

Stomach (ST) 34, ST 35, ST 36

Spleen (SP) 9, SP 10

BL 40 KID 10

GB 33, GB 34

LIV 8

Extrapoint (EX) Heding, EX Xiyan

\section{Distant points}

Choice of at least 2 local acupoints from the following permitted sample: SP 4, SP 5, SP 6

SI 6

BL 20 BL 57, BL 58, Bl 60, BL 62

KID 3

Additional points of the knee (body and microsystem acupuncture) can be chosen.

Bilateral osteoarthrosis: Local and distant points must be acupunctured bilaterally.

The total number of needles must not exceed 20 .

Unilateral osteoarthrosis of the knee: Procedere (uni- or bilateral acupuncture) depends on the assessment and experience of the physician. When acupuncture is unilateral, number of needles must not exceed 16.

[22]; (2) a scale for assessing the emotional aspects of pain ('Schmerzempfindungs-Skala', SES) [23]; (3) the depression scale ADS [24]; and (4) the German version of the SF-36 to assess health-related quality of life [25]. Further secondary outcome parameters are: (1) the number of days with pain medication in the weeks 4-8 after randomization (documented in a diary), (2) global assessment of therapeutic effectiveness measured by the questionnaire on overall treatment effect [26] at week 8
Table 3. Minimal points used in both ART trials; one 'cun' is defined according to the rules of traditional Chinese medicine as the width of the interphalangeal joint of patient's thumb

MA point 'Deltoideus': in the middle of the insertion line of M. deltoideus

(LI 14) and Acromion

MA point 'Upper Arm': 2 cun laterally (radial) of LU 3

MA point 'Forearm': 1 cun ulnar of the proximal third of the line between heart (HE) 3 and $\mathrm{HE} 7$

MA point 'Scapula': 1 cun laterally of the lower scapular edge

MA point 'Spina Iliaca': 2 cun above spina iliaca anterior superior in vertical line to the arch of left ribs

MA point 'Back I': 5 cun laterally of the spine of lumbar vertebrum IV

MA point 'Back II': 5 cun laterally of the spine of lumbar vertebrum V

MA point 'Upper Leg I': 6 cun above the upper edge of the patella

(between the spleen and stomach meridian)

MA point 'Upper Leg II': 4 cun above the upper edge of the patella

MA point 'Upper Leg III': 2 cun dorsally of GB 31 (avoidance of bladder meridian)

In Gon-ART the MA point 'Upper Leg II' is replaced with the following point:

MA point 'Lower Leg': 2 cun laterally (fibulary) and 1 cun caudal of BL 56.

after randomization, and (3) the number of side effects and adverse effects.

In order to test the blinding to treatment, patients fill in a questionnaire after the third acupuncture session in order to assess the credibility of the respective treatment methods [27]. At the end of the study, patients are asked whether they believe that they have received acupuncture following the principles of Chinese medicine or the other type of acupuncture. For each session, physicians are asked to report whether side effects or adverse effects occur. In addition, the patients are asked to report side effects in the above-mentioned questionnaires both at the end of 8 and 26 weeks after randomization (fig. 1). Drop-outs and withdrawals and the respective reasons are documented.

Furthermore, we employ the Mainz Pain Staging System (MPSS) (Mainzer Stadieneinteilung) [28], a physician-based questionnaire used to characterise the chronicity of disease.

\section{Statistics}

Analyses will be performed for two populations: (1) an intention to treat population with all patients randomized and (2) a per protocol population including only patients with no major protocol deviations. All data will be analyzed descriptively.

Confirmatory testing of the main outcome measure and all main analyses will be based on the intention to treat population. A hierarchical test procedure will be performed in order to maintain a global significance level of $\alpha=5 \%$. First, the two-sided null hypothesis to be tested is $\mathrm{H}_{0,1}$ : acupuncture $=$ waiting list. Only if the test results are significant will a second test for $\mathrm{H}_{0,2}$ : acupuncture = minimal acupuncture be performed.

For each of the hypotheses, Student's t-test and $\alpha=5 \%$ will be used. The waiting list group will be included in the main analysis only until week 8 after randomisation. In the event of relevant imbalances between the randomized treatment groups, statistical analysis will be performed using a covariance model (ANCOVA) with the factor treatment group and the imbalanced covariates.

The studies are powered to detect with $80 \%$ power a difference of 8 score points in the respective primary target variables (i.e. WOMAC score [29] for the osteoarthritis trial and $10 \mathrm{~mm}$ on the VAS for the low back pain trial) between the acupuncture and minimal acupuncture groups, assuming a standard deviation of 17 score points and $22.5 \mathrm{~mm}$, respectively. For sample size calculations, a decrease in the WOMAC score from baseline 
50 to 25 after treatment ( $50 \%$ reduction) in the acupuncture and 50 to 33 (33\% reduction) in the minimal acupuncture group was assumed for the osteoarthritis trial. For the low back pain trial, the respective assumptions were a 60 to $30 \mathrm{~mm}(50 \%)$ reduction on the VAS in the acupuncture group, and a 60 to $40 \mathrm{~mm}(33 \%)$ reduction in the minimal acupuncture group. The waiting list control group will be included in the main analysis only until week 8 after randomization.

\section{Discussion}

Compared to previous studies of acupuncture in the treatment of low back pain and osteoarthritis of the knee $[5,12]$ the ART studies have a much larger number of participating acupuncturists and will include significantly more patients.

In acupuncture research, it would seem desirable to take into consideration more objective endpoints, such as changes in laboratory parameters. However, in the case of low back pain and osteoarthritis of the knee, the subjective target parameters used in the present studies are well established and of clear clinical relevance [30].

When practiced according to the principles of Traditional Chinese Medicine (TCM), acupuncture is an individualized therapy [31]. In the ART studies, we use a semi-standardized treatment protocol, similar to a number of other studies in the past [e.g. 32]. In all patients, we require that a number of predefined 'basic points' be used, but allow physicians to add further points based, for example, on an individualized syndrome diagnosis made according to TCM diagnostic methods, or on clinical experience. The predefined 'basic points' were carefully selected in a consensus process with experts from two German acupuncture societies while taking the principles of TCM into account. The main reason for choosing a semi-standardized treatment was to foster transparency and a certain degree of replicability without losing individualization. The treatment protocol chosen is not necessarily representative of the practice of acupuncture in Germany or elsewhere, because there are many different approaches to perform acupuncture. Thus, it is important to emphasize that the ART studies, like all other studies on acupuncture, do not investigate the effectiveness of acupuncture in general, but rather the effectiveness of specific acupuncture interventions.

For methodological reasons, we decided not to include moxibustion - an additional thermal stimulation on the skin using Artemisia vulgaris - in the treatment protocols of the ART studies, as the potential specific and non-specific effects of this therapy cannot be separated from the effects of acupuncture. This decision was unproblematic for osteoarthrosis of the knee, migraine, and tension-type headache, because moxibustion is rarely used to treat these conditions. However, this was not the case with low back pain, as moxibustion is widely used in the treatment of this particular condition. Because of this, we have instructed our physicians not to include patients in the low back pain study if their condition warrants treatment with moxibustion.
A number of the available acupuncture studies have been criticised for unclear or insufficient quality of acupuncture [33]. We have used explicit and rigid criteria to ensure that the physicians in the study have a high level of education and practical experience. Nevertheless, it must be pointed out that the education of German acupuncturists is varied. All acupuncturists in the ART studies are physicians who have completed their primary training in acupuncture in Western Europe and have spent little or no time training in China.

The Federal Committee has explicitly requested that research be conducted on body acupuncture compared with 'placebo' or 'sham' acupuncture. The problems of ensuring adequate control treatment in acupuncture trials are discussed elsewhere [2].

The ART studies are part of two major evaluation projects ('Modellvorhaben Akupunktur') which also include several pragmatic randomized trials and large observational studies with several hundreds of thousands of patients treated by over 10,000 physicians in Germany [34, 35]. These observational studies evaluate patient characteristics, interventions, and outcomes under routine conditions. In this trial, we will assess especially those results related to the efficacy, side effects, and socio-economic aspects of acupuncture treatment under real-life conditions. Together with a third 'Modellvorhaben Akupunktur' [36], the results of these evaluation projects will provide a sound basis for the decision on whether or not acupuncture will be covered by health insurance companies in Germany. By early 2004, the final results of the ART studies should be available.

In conclusion, the Acupuncture Randomized Trials belong to the largest and most rigorous studies of acupuncture to be conducted thus far. We hope that the results of these studies will represent a major step forward in the scientific evaluation of the effectiveness of acupuncture for all indications.

\section{Acknowledgments}

We would like to thank Dr. U. Walther and Prof. Dr. H. Zippel, Department of Orthopaedics, Charité Hospital, Humboldt University of Berlin, for advice on the indications low back pain and osteoarthrosis of the knee, and Prof. A. Neiss, Institute of Medical Statistics and Epidemiology, Technische Universität München, PD Dr. M. Kulig, Institute of Social Medicine, Epidemiology, and Health Economics, Charité Hospital, Humboldt University of Berlin, and Prof. K. Wegscheider, Biometrie und Statistik $\mathrm{GmbH}$, Berlin, for advice on randomization procedures and statistics. We thank Dr. M. Hammes, Department of Neurology, Technische Universität München, for advice in the consensus process on treatment strategies.

We thank the following acupuncture experts for participation in the consensus process:

German Medical Acupuncture Association (DÄGfA) - Dr. J. Bachmann, Hattingen; Dr. N. Behrens, München; Dr. E. Berr, München; Dr. H. Garten, München; Dr. J. Gelditsch, Baierbrunn; G. Huemer, Nussdorf; H. Luxenburger, Aachen; Dr. P. Novoa-Lill, München; Dr. W. Maric-Oehler, Bad Homburg; Prof. Dr. T. Ots, Graz (Österreich); Dr. O. Mastalier, Oberaudorf; J. Mücher, Bremen; Dr. H. Ogal, Brunnen (Schweiz); Dr. R. Pothmann, Oberhausen; Dr. G. Peters, Hankensbüttel; Dr. K.D. Platsch, 
Prien; Dr. A. Pollmann, Hamburg; Dr. H. Rausch, Oberhausen; Dr. A Rubach, München; Dr. H. Rüdinger, Hamburg; Dr. A. Schneider, Heidelberg; S. Schmitz, Hufschlag; Dr. U. Siedentopp, Kassel; Dr. A. Steveling, Bochum; Dr. W. Stör, Icking; Dr. C. Vogel, Mainz; A. Volmer, Ahrensburg; Dr. H.J. Weise, Rheinfelden
International Society for Chinese Medicine (SMS) - Dr. C.H. Hempen, München; H. Leonhardy, München; Dr. R. Nögel, München.

The ART studies are funded as a part of the 'Modellvorhaben der Techniker Krankenkasse und der dem Modellvorhaben beigetretenen Krankenkassen' and the 'Modellvorhaben der Ersatzkassen'.

\section{References}

1 Bekanntmachung einer Änderung der Richtlinien über die Bewertung ärztlicher Untersuchungs- und Behandlungsmethoden gemäß $§ 135$ Abs. 1 Fünftes Buch Sozialgesetzgebung vom 16. Oktober 2000. Bundesanzeiger Januar 2001.

2 Melchart D, Linde K, Streng A, Reitmayr S, Hoppe A, Brinkhaus B, Becker-Witt C, Wagenpfeil S, Pfaffenrath V, Hammes M, Willich SN, Weidenhammer W: Acupuncture Randomized Trials (ART) in patients with migraine or tension-type headache - design and protocols. Forsch Komplementärmed Klass Naturheilkd 2003;10:179-184.

3 Ernst E, White A: Acupuncture for back pain. A meta-analysis of randomized controlled trials. Arch Intern Med 1998;158:2235-2241.

4 Ernst E, White A, Wider B: Akupunktur bei Rückenschmerzen. Schmerz 2002;16:129-139.

5 van Tulder MW, Cherkin DC, Berman B, Lao L, Koes BW: The effectiveness of acupuncture in the management of acute and chronic low back pain. Spine 1999;24:1113-1123.

6 Smith LA, Oldman AD, McQuay HJ, Moore RA Teasing apart quality and validity in systematic reviews: An example from acupuncture trials in chronic neck and back pain. Pain 2000;86:119-132.

7 Carlsson CP, Sjolund BH: Acupuncture for chronic low back pain: A randomized placebo-controlled study with long-term follow-up. Clin J Pain 2001; 17:296-305.

8 Cherkin DC, Eisenberg D, Sherman KJ, Barlow W, Kaptchuk TJ, Street J, Deyo RA: Randomized trial comparing traditional Chinese medical acupuncture, therapeutic massage, and self-care education for chronic low back pain. Arch Intern Med 2001; 161:1081-1088.

9 Leibing E, Leonhardt U, Koster G, Goerlitz A, Rosenfeldt JA, Hilgers R, Ramadori G: Acupuncture treatment of chronic low-back pain - a randomized, blinded, placebo-controlled trial with 9month follow-up. Pain 2002;96:189-196.

10 Molsberger AF, Mau J, Pawelec DB, Winkler J: Does acupuncture improve the orthopedic management of chronic low back pain - a randomized, blinded, controlled trial with 3 months follow up. Pain 2002:99:579-587.

11 Hiethard FU, Pfeil J: Degenerative Gelenkserkrankungen. Orthopädie, 3. Aufl. Stuttgart, Hippokrates, 1997, pp 190-196.
12 Ezzo J, Hadhazy V, Birch S, Lao L, Kaplan G, Hochberg M, Berman B: Acupuncture for osteoarthritis of the knee. A systematic review. Arthritis Rheum 2001;44:819-825.

13 Tillu A, Tillu S, Vowler S: Effect of acupuncture on knee function in advanced osteoarthritis of the knee: A prospective, non-randomised controlled study. Acupunct Med 2002;20:19-21.

14 Machin D, Campbell MJ, Fayers PM, Pinol AY: Sample Size Tables for Clinical Studies. Oxford, Blackwell, 1997.

15 Kellgren JH, Lawrence JS: Radiological assessment of osteo-arthrosis: Ann Rheum Dis 1957;16: 494-502.

16 Kessler S, Guenther KP, Puhl W: Scoring prevalence and severity in gonarthritis: The suitability of the Kellgren and Lawrence scale. Clin Rheumatol 1998;17:205-209.

17 Huskisson EC: Measurement of pain. Lancet 1974; 2:1127-1131.

18 Kohlmann T, Raspe H: Der Funktionsfragebogen Hannover zur alltagsnahen Diagnostik der Funktionsbeeinträchtigung durch Rückenschmerzen (FFbH-R). Rehabilitation 1996;35:1-8.

19 Barr S, Bellamy N, Buchanan W, Chalmers A, Ford PM, Kean WF, Kraag GR, Gerecz-Simon J, Campbell J: A comparative study of signal versus aggregate methods of outcome measurement based on the WOMAC osteoarthritits index. J Rheumatol 1994;21:2106-2112.

20 Bellamy N, Buchanan WW, Goldsmith CH, Campbell J, Stitt LW: Validation study of WOMAC: A health status instrument for measuring clinically important patient-relevant outcomes following total hip and knee arthroplasty in osteoarthritis. J Orthop Rheumatol 1988;1:95-108.

21 Stucki G, Meier D, Stucki S, Michel BA, Tyndall AG, Dick W, Theiler R: Evaluation einer deutschen Version des WOMAC (Western Ontario und McMaster Universities) Arthroseindex. Z Rheumatol 1996;55:40-49.

22 Dillmann U, Nilges P, Saile H, Gerbershagen HU: Behinderungseinschätzung bei chronischen Schmerzpatienten. Schmerz 1994;8:100-110.

23 Geissner E, Schulte A: Die SchmerzempfindungsSkala (SES). Weinheim, Beltz, 1996.
24 Hautzinger M, Bailer M: Allgemeine Depressionsskala (ADS). Die deutsche Version des CES-D. Weinheim, Beltz, 1991.

25 Bullinger M, Kirchberger I: SF-36 Fragebogen zum Gesundheitszustand. Göttingen, Hogrefe, 1998

26 Juniper EF, Guyatt GH, Willan A, Griffith LE: Determining a minimal important change in a disease-specific quality of life questionnaire. J Clin Epidemiol 1994:47:81-87.

27 Vincent C: Credibility assessments in trials of acupuncture. Complement Med Res 1990;4:8-11.

28 Gerbershagen HU: Organisierte Schmerzbehandlung. Eine Standortbestimmung. Internist 1986;27: 459-469.

29 Berman BM, Singh BB, Lao L, Langenberg P, Li H, Hadhazy V, Bareta J, Hochberg M: A randomized trial of acupuncture as an adjunctive therapy in osteoarthritis of the knee. Rheumatology 1999; 38:346-354.

30 Creamer P, Hochberg MC: Osteoarthritis. Lancet 1997;350:503-508.

31 Cheng Xinnong: Chinese Acupuncture and Moxibustion. Beijing, China, Foreign Languages Press, 1987.

32 Joos S, Schott C, Zuo H, Daniel V, Martin E: Immunomodulatory effects of acupuncture in the treatment of allergic asthma: A randomized controlled trial. J Altern Complement Med 2000;6: 519-525.

33 Stux G, Hammerschlag R (eds.): Clinical Acupuncture. Scientific Basis. Berlin, Springer, 2001.

34 Becker-Witt C, Brinkhaus B, Jena S, Löbel L, Menke M, Willich SN: Modellvorhaben Akupunktur der Techniker Krankenkasse und der dem Modellvorhaben beigetretenen Krankenkassen. Z Arztl Fortbild Qualitatssich 2003;97 (in print).

35 Melchart D, Streng A, Reitmayr S, Hoppe A, Weidenhammer W, Linde K: Programm zur Evaluation der Patientenversorgung mit Akupunktur (PEPAK) - Die wissenschaftliche Begleitung des Modellvorhabens der Ersatzkassen. Z Arztl Fortbild Qualitatssich 2003;97 (in print).

36 Molsberger A, Diener HC, Krämer J, Michaelis J, Schäfer H, Trampisch HJ, Victor N, Zenz M: GER AC-Akupunkturstudien. Modellvorhaben zur Beurteilung der Wirksamkeit. Dtsch Ärztebl 2002;99: 1819-1821 